\title{
BMJ Open Microablative fractional radiofrequency for the genitourinary syndrome of menopause: protocol of randomised controlled trial
}

Ayane Cristine Alves Sarmento, ${ }^{1}$ Fabíola S Fernandes, ${ }^{2}$ Ana Paula Ferreira Costa, ${ }^{1}$ Kleyton Santos Medeiros, ${ }^{1}$ Janaina Cristina Crispim, ${ }^{2}$

Ana Katherine Gonçalves (1) 1,3

To cite: Sarmento ACA, Fernandes FS, Costa APF, et al. Microablative fractional radiofrequency for the genitourinary syndrome of menopause: protocol of randomised controlled trial. BMJ Open 2021;11:e046372. doi:10.1136/ bmjopen-2020-046372

- Prepublication history for this paper is available online. To view these files, please visit the journal online (http://dx.doi. org/10.1136/bmjopen-2020046372).

Received 31 October 2020 Accepted 06 June 2021

\section{Check for updates}

\section{(c) Author(s) (or their} employer(s)) 2021. Re-use permitted under CC BY-NC. No commercial re-use. See rights and permissions. Published by BMJ.

${ }^{1}$ Health Sciences Postgraduate Program, Federal University of Rio Grande do Norte, Natal, Brazil

${ }^{2}$ Department of Clinical Analysis and Toxicology, Federal University of Rio Grande do Norte, Natal, Brazil

${ }^{3}$ Department of Obstetrics and Gynaecology, Federal University of Rio Grande do Norte, Natal, Brazil

\section{Correspondence to} Dr Ana Katherine Gonçalves; anakatherine_ufrnet@yahoo. com.br

\section{ABSTRACT \\ Introduction Menopause is a physiological and} progressive phenomenon secondary to decreased ovarian follicular reserve. These changes have consequences: vaginal dryness, dyspareunia, discomfort, burning and irritation, vulvovaginal pruritus, dysuria and increased frequency of genitourinary infections. The therapy more suitable for vaginal symptoms in postmenopause yet is the use of a topical hormone. However, the prescription of topical oestrogens should also be avoided in women with a history of breast cancer, oestrogen-sensitive tumours and thromboembolism, emphasising the necessity of alternative treatments. Recently, physical methods, such as laser and radiofrequency (RF), in their non-ablative, ablative and microablative forms have been used in the vaginal mucosa to promote neocolagenesis and neoelastogenesis. This randomised study aims to compare the efficiency of microablative fractional RF (MAFRF) treatment with vaginal oestrogens and no treatment. Methods and analyses This randomised, controlled clinical intervention trial with an open label design comparing the treatment of MAFRF with vaginal oestrogens and no treatment. Four important moments were considered to evaluate treatment results $(\mathrm{T} 0, \mathrm{~T} 1$, T2 and T3). The primary outcome includes vulvovaginal atrophy (vaginal pain, burning, itching, dryness, dyspareunia and dysuria), and the secondary outcomes will be sexual function, vaginal health (epithelial integrity, vaginal elasticity, moisture, fluid volume and vaginal $\mathrm{pH}$ ) and quality of life.

Ethics and dissemination Due to the nature of the study, we obtained approval from the ethics committee. All participants must sign an informed consent form before randomisation. The results of this study will be published in peer-reviewed journals. The data collected will also be available in a public repository of data.

Trial registration number RBR-94DX93.

\section{INTRODUCTION}

Menopause is a physiological and progressive phenomenon, secondary to decreased ovarian follicular reserve. Oestrogen deficiency is responsible for the mucosa vulvovaginal thinning of the squamous epithelium,
Strengths and limitations of this study

- The latter is the first randomised controlled trial comparing the microablative fractional radiofrequency with the golden standard (vaginal oestrogen).

- Inclusion criteria allow homogeneity of subjects and less risk of bias.

- Blinding of assessors and standardisation of protocols enhance this trial's internal validity.

- The study will be performed among postmenopausal women; thus, its results may not be generalisable to other populations.

a decrease in the number of collagen and elastin fibres, impaired cell function, reduction in the number of vessel, changes in vaginal $\mathrm{pH}$, and in flora commensal. ${ }^{1}$

These changes have functional consequences, being vaginal dryness, dyspareunia, sensations of discomfort, burning and irritation, vulvovaginal pruritus, dysuria and increased frequency of genitourinary infections. ${ }^{1}$ The term genitourinary syndrome of menopause (GSM) was defined in 2014 at a North American consensus conference, where all the disabling symptoms of the urogenital sphere secondary to menopause were more fully and objectively identified. ${ }^{2}$

The therapy more suitable for vaginal symptoms in post menopause is the use of the topical hormone, which promotes the renovation of the epithelium and vaginal flora, and improves the vulvovaginal atrophy (VVA) symptoms. ${ }^{3}$ Besides that, the use of low-dose vaginal oestrogen has been demonstrated to be superior to systemic therapy for improvement of VVA. ${ }^{4}$ However, a major limitation is associated with low adherence to treatment, due to multiple and inconvenient selfapplications and increased vaginal discharge. We cannot fail to emphasise that the 
prescription of topical oestrogens should be avoided in women with a history of breast cancer, oestrogen-sensitive tumours and thromboembolism. For these reasons, it is necessary to search for alternatives to treatment. ${ }^{5}$

The use of fractional microablative $\mathrm{CO}_{2}$ laser therapy was approved in 2014 by the Food and Drug Administration, but only for genitourinary surgery. Considering the context of the treatment of GSM, in order to avoid hormonal interventions, the microablative fractional $\mathrm{CO}_{2}$ laser, or the non-ablative vaginal erbium YAG (yttriumaluminum-garnet) laser can be considered. Recently, studies showed that the use of fractional $\mathrm{CO}_{2}$ laser in the treatment of VVA was beneficial, effective and safe. The positive effects on VVA symptoms improved not only the quality of life, but also the aspect of sexual pain and other dimensions of women's sexual response, such as desire, initiative and receptivity to their sexual partner. ${ }^{6-8}$ Similar results have been observed with the use of YAG laser treatment. Application of Er:YAG laser is associated with an improvement in vaginal atrophy, and such treatment induced a significant decrease in the Visual Analogue Scale (VAS), an increase of Vaginal Health Index (VHI) and a significant improvement in urinary incontinence (UI). ${ }^{9} 10$ Beyond the laser, other non-ablative electromagnetic energy, such as radiofrequency (RF), are being considered for this indication. ${ }^{11}$

$\mathrm{RF}$ is a technique that involves cutting and coagulating biological tissues by using a high-frequency alternating current, which instantly raises the intracellular temperature to $100^{\circ} \mathrm{C}$, thus determining cellular membrane expansion and rupture. By reaching the frequency of 4000000 cycles/s $(4 \mathrm{MHz})$, the FM radio frequency is obtained-this feature gives rise to the name RF electrosurgery. Microablative fractional RF (MAFRF) is a new procedure that uses random energy in a fractionation system that observes the thermal relaxation of the tissue at a certain time. Energy fractionation consists of energy distribution at equidistant points, producing microscopic columns of thermal injuries in the epidermis and upper dermis, resulting in microscopic columns of treated tissue and intervening areas of untreated skin, which, in turn, achieve faster re-epithelialisation. ${ }^{12} 13$

We can consider that the current literature lacks still needs research regarding the use of RF for the treatment of GSM, as most publications evaluate only the use of laser. The few studies that have been performed on RF, are mostly restricted to results on sexual function and quality of life. There are no results about the clinical evaluations, such as $\mathrm{pH}$, cellularity and vaginal flora when using RF, and that is exactly our proposal.

\section{Objectives}

We aim to investigate the therapeutic effect of vaginal MAFRF in the GSM. We postulate that MAFRF could promote the improvement of VVA. Furthermore, it could improve sexual function, vaginal health and quality of life in postmenopausal women.

\section{METHODS AND ANALYSIS}

This protocol will adhere to the Standard Protocol Items for Randomised Trials ${ }^{14}$ and Consolidated Standards of Reporting Trials (CONSORT) statements. ${ }^{15}$

\section{Trial design}

The study is a randomised protocol, controlled clinical intervention trial with a single-blind design comparing treatment with MAFRF with vaginal oestrogens as well as no treatment.

\section{Population}

Participant recruitment is currently ongoing at a gynaecological unit of a public university hospital. Patients who fulfil the inclusion criteria and who sign the free and informed consent forms will enter the screening period. Patients who meet the exclusion criteria will be excluded before randomisation.

\section{Eligibility criteria and recruitment}

The treatments will be conducted at one of the biggest gynaecological units of a public university hospital in Brazil. Participants will be sought via referrals from gynaecologist physicians that attend this hospital. The study will include healthy postmenopausal women (55-65 years old, with whom at least 12 months have passed since the last menstrual period or bilateral oophorectomy), who are still sexually active, with GSM, plasma gonadotropin and presenting serum estradiol levels in the postmenopausal range (follicle stimulating hormone $>40 \mathrm{U} / \mathrm{L}$; estradiol $<25 \mathrm{pg} / \mathrm{mL}$ ) as well as negative Papanicolaou smear for cervical cancer precursor cells. Women who have used any form of hormonal (systemic or local) therapy in the last 6 months, lubricants or vaginal moisturisers in the past month, suffering from active genital infections (diagnosis by Gram stain and multiplex PCR) and any disease that would interfere with following the protocol will be excluded. Figure 1 shows the study flow.

\section{Interventions}

The MAFRF will be performed according to the technique described by Kamilos and Borelli. ${ }^{13}$ For the procedure, the Wavetronic 6000 Touch device will be used with the Megapulse HF FRAXX system (Loktal Medical Electronics, São Paulo, Brazil), equipped with an electronic circuit of energy fractionation, connected to a vaginal pen with 64 microneedles, $200 \mu$ in diameter and $1 \mathrm{~mm}$ in length, mounted on a Teflon body and divided into an eight column matrix with eight needles in each. ${ }^{13}$

In the vestibule and vaginal opening, $10 \%$ lidocaine spray will be applied $3 \mathrm{~min}$ before the procedure. Three applications will be realised in the vagina/vaginal introitus, with intervals of 30 days. A sequential application will be performed on the vaginal walls under direct vision. For the post-treatment care, the use of $5 \%$ dexpanthenol solution in the vaginal opening will be recommended two to three times a day, for $2-5$ days, with no intercourse for 10 days. ${ }^{13}$ The procedure will be performed in the outpatient clinic by an experienced gynaecologist, and a single 


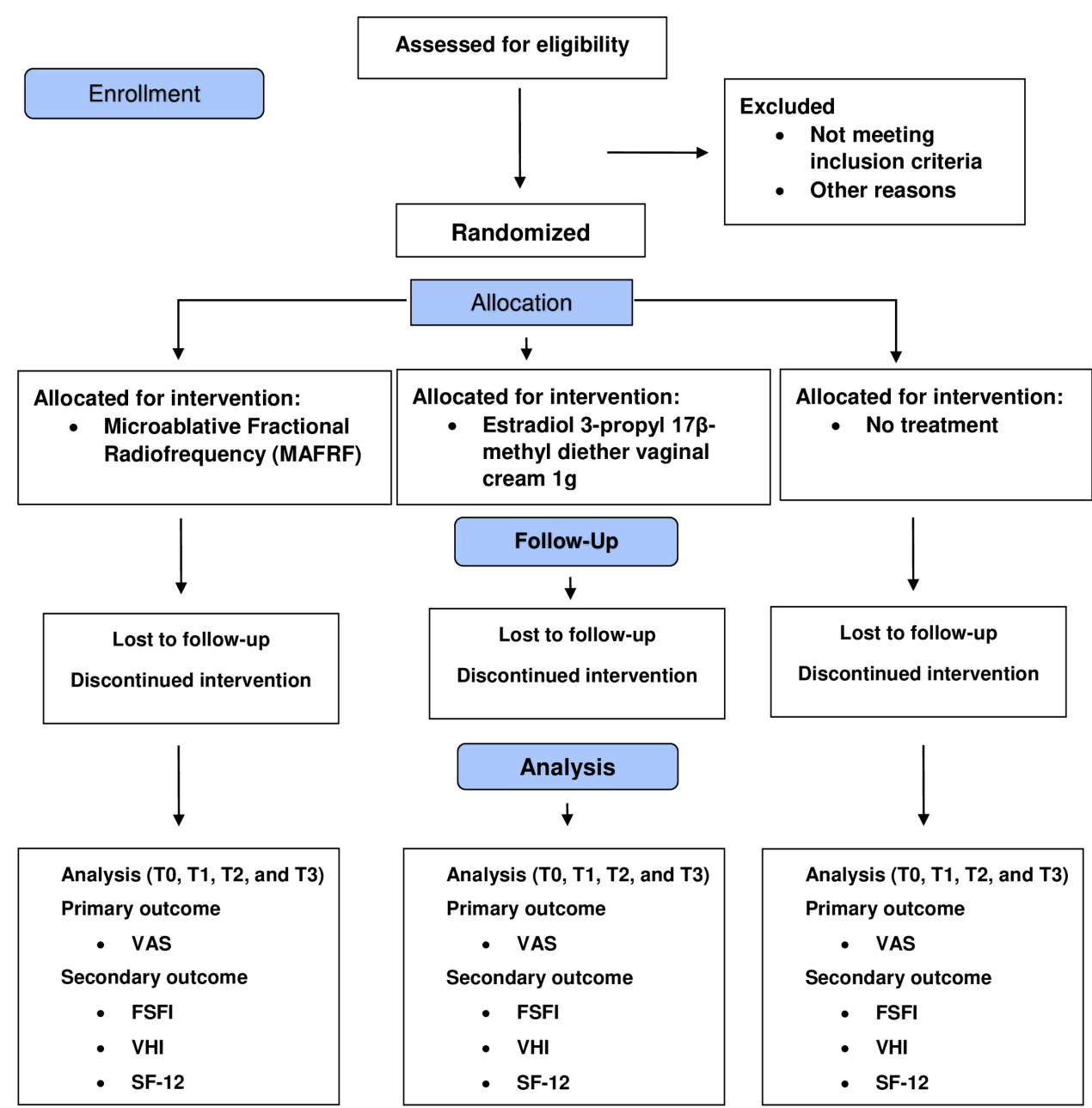

Figure 1 CONSORT 2010 flow diagram. CONSORT, Consolidated Standards of Reporting Trials; FSFI, Female Sexual Function Index; SF-12, Short Form 12; VAS, Visual Analogue Scale; VHI, Vaginal Health Index.

gynaecologist will supervise the whole process for the entire period of the research.

The patients from the group with oestrogen will be instructed to use promestriene (estradiol 3-propyl $17 \beta$-methyl diether) vaginal cream, $1 \mathrm{~g}$ corresponding to the use of the filled applicator up to the ring mark, twice a week, for 3 months. ${ }^{16-18}$

The patients who did not receive any intervention will be instructed to attend the consultation for follow-up according to what was established in the study protocol.

\section{Questionnaire}

In the first query, the women will answer a standardised questionnaire with information on demographic characteristics, including age, time menopause, skin colour, schooling and socioeconomic classification.

\section{Outcomes}

Four relevant time points will be considered for evaluating the results based on a previous study: $:^{13}$ baseline (T0), 30 days after the first application (T1), 30 days after the second application (T2) and 30 days after the third RF application (T3).

The primary outcome includes VVA using the 11-point VAS. The VAS associate symptoms (vaginal pain, burning, itching, dryness, dyspareunia and dysuria). ${ }^{19-22}$

Secondary outcomes will be sexual function, vaginal health (epithelial integrity, vaginal elasticity, moisture, fluid volume and vaginal $\mathrm{pH}$ ) and quality of life. Female sexual function will be evaluated using the validated Portuguese version of the Female Sexual Function Index (FSFI). FSFI is a brief scale for assessing sexual function in women. It is a written test with six subscales and one sum of scores that measure the degree of desire, excitement, lubrication, orgasm, satisfaction, and pain (dyspareunia). ${ }^{23-25}$

The Short Form 12 (SF-12) is a self-reported outcome measure assessing the impact of health on an individual's everyday life. The SF-12 assesses the physical component summary (PCS12) and mental component summary (MCS12) scores. It is often used as a quality of 
Table 1 Outcome measurements

\section{Outcome}

measurements Explanation

Time points for assessment

\begin{tabular}{|c|c|c|}
\hline VAS & $\begin{array}{l}\text { VAS evaluates the change in six categories of symptoms commonly associated with } \\
\text { vulvovaginal atrophy: vaginal pain, burning, itching, dryness, dyspareunia and dysuria. } \\
\text { VAS will be scored on an 11-point scale for each symptom, with } 0 \text { being the lowest } \\
\text { level (none) and } 10 \text { being the highest (extreme). }{ }^{19-22}\end{array}$ & $\mathrm{~T} 0, \mathrm{~T} 1, \mathrm{~T} 2$ and T3 \\
\hline FSFI & $\begin{array}{l}\text { The FSFI evaluates six subscales and one sum of scores that measure the degree } \\
\text { of desire, excitement, lubrication, orgasm, satisfaction and pain (dyspareunia). The } \\
\text { scores of the subscales are corrected and added up, resulting in a final score. Final } \\
\text { scores can range from } 2 \text { to } 36 \text {. Higher scores indicate a better degree of sexual } \\
\text { function. }{ }^{23-25}\end{array}$ & $\mathrm{~T} 0, \mathrm{~T} 1, \mathrm{~T} 2$ and $\mathrm{T} 3$ \\
\hline VHI & $\begin{array}{l}\text { VHI scores of vaginal moisture, vaginal fluid volume, vaginal elasticity, } \mathrm{pH} \text { and vaginal } \\
\text { epithelial integrity on a scale of } 1 \text { (most inferior) to } 5 \text { (best) will be found. Vaginal } \\
\text { moisture is an assessment of the appearance or consistency of the secretions that } \\
\text { line the vagina. Vaginal elasticity is a measurement of the vaginal tissue's ability to } \\
\text { stretch at the examiner's touch. Epithelial integrity takes into account colour, thickness } \\
\text { and the absence of vaginal bleeding. The lower the score, the higher the atrophy. The } \\
\text { sum of the values of the evaluated parameters results in the total vaginal health score. } \\
\text { When the overall score is less than } 15 \text {, the vaginal mucosa is considered atrophic. }{ }^{1927}\end{array}$ & $\mathrm{~T} 0, \mathrm{~T} 1, \mathrm{~T} 2$ and $\mathrm{T} 3$ \\
\hline SF-12 & $\begin{array}{l}\text { The SF-12 provides accurate and efficient information to assess physical and } \\
\text { mental health. It includes eight dimensions as the initial SF-36 instrument: general } \\
\text { health perceptions (one item), physical functioning (two items), role limitations due } \\
\text { to physical problems (two items), bodily pain (one item), vitality (one item), social } \\
\text { functioning (one item), role limitations due to emotional problems (two items) and } \\
\text { mental health (two items). The composite physical (PCS) and mental health (MCS) } \\
\text { scores are computed using the scores of the } 12 \text { items, ranging from } 0 \text { to } 100, \text { where } \\
\text { zero reflects the lowest health level and } 100 \text { the highest level. }{ }^{20-22} 26\end{array}$ & $\mathrm{~T} 0, \mathrm{~T} 1, \mathrm{~T} 2$ and $\mathrm{T} 3$ \\
\hline
\end{tabular}

FSFI, Female Sexual Function Index; MCS, mental component summary; PCS, physical component summary; SF-12, Short Form 12; VAS, Visual Analogue Scale; VHI, Vaginal Health Index.

life measure. ${ }^{20-22} 26$ Vaginal health will be evaluated per Vaginal Health Index (VHI). The VHI consists of the clinical analysis during the specular examination of five parameters and is graded from 1 to 5. The sum of the values of the parameters evaluated results in the total vaginal health score. ${ }^{19} 27$ The evaluation of the results is described in table 1.

All possible adverse effects will be recorded and qualified during the period of treatment using questionnaires developed for this protocol. The adverse events will be reported in the results section of the manuscript and will be discussed. Any breaches of confidentiality, study protocol or adverse events attributable to this study will be reported to the research ethics committees.

\section{Follow-up}

Data will be recorded during the follow-up period according to the multiple time points. The details are shown in table 2.

Period 1: Screening time (day 0): before treatment.

Period 2: Intervention time (T1-T3): data will be recorded every 7 and 30 days during follow-up.

Period 3: The time after the intervention (within 1 year after treatment): follow-up at 1 year for long-term results.

\section{Sample size}

Therefore, with an equal 1:1:1 allocation rate, according to the results of a pilot study, where data came from 55 volunteers, ${ }^{28}$ accepting an alpha of 0.05 and a beta risk

Table 2 Schedule of enrolment, interventions, assessments and data collection

\begin{tabular}{|c|c|c|c|c|c|}
\hline \multirow[b]{3}{*}{ Time point } & \multicolumn{5}{|l|}{ Study period } \\
\hline & \multirow{2}{*}{$\begin{array}{l}\begin{array}{l}\text { Enrolment/ } \\
\text { baseline }\end{array} \\
\text { T0 }\end{array}$} & \multicolumn{3}{|c|}{ Intervention } & \multirow{2}{*}{$\begin{array}{l}\text { Follow-up } \\
1 \text { year }\end{array}$} \\
\hline & & $\mathrm{T} 1$ & T2 & T3 & \\
\hline Enrolment & $x$ & & & & \\
\hline Eligibility screen & $x$ & & & & \\
\hline Informed consent & $x$ & & & & \\
\hline Randomisation & $x$ & & & & \\
\hline \multicolumn{6}{|l|}{ Interventions } \\
\hline $\begin{array}{l}\text { Microablative } \\
\text { fractional } \\
\text { radiofrequency }\end{array}$ & & $x$ & $x$ & $x$ & \\
\hline Vaginal oestrogens & & $x$ & $x$ & $x$ & \\
\hline No treatment & & $x$ & $x$ & $x$ & \\
\hline \multicolumn{6}{|l|}{ Assessments } \\
\hline General condition & & & & & $\mathrm{X}$ \\
\hline
\end{tabular}


$<0.2$ in a bilateral contrast, assuming a patient attrition rate at follow-up of approximately $15 \%-20 \%$, the total sample size needed isestablished at 198 patients, 66 in each group (66 in the MAFRF group, 66 with vaginal oestrogens and 66 in the no treatment).

\section{Randomisation and allocation concealment}

Eligible participants who provide written consent will be randomised into the Software Research Randomizer programme. Randomisation will be by block (1:1:1). Patients will be randomised into three intervention groups (MAFRF group, vaginal oestrogens group and no treatment group). In order to ensure allocation concealment, an off-site randomisation schedule will be used. The randomisation schedule will be prepared in advance by a researcher at the Federal University of Rio Grande do Norte (UFRN), who will have no contact with any participants throughout the trial and will not be involved in the recruitment, screening, assessment, enrolment or treatment process. To enrol a participant, the primary researcher will email the consenting participant's name to the researcher at the UFRN. These details will be entered into the allocation spreadsheet, and the next treatment allocation and participant identification number will be emailed to the researcher.

\section{Blinding}

Participants and the group researchers cannot be blind to arm allocation because of the features of the interventions (MAFRF and vaginal oestrogens). However, the researchers who will evaluate the outcomes will be blinded to which arm comprises each intervention and any other sociodemographic information that might facilitate the identification of the intervention group.

\section{Data management}

The qualification of the researchers involved in research is crucial that ensure the quality of the clinical trial. The researchers should understand the specific contents of the protocol. Data collection will be performed by experienced staff using an online electronic data system. The quality of the data management will be checked by the reliability, controlled access and traceability of the system. Data management will include baseline characteristics (demographics, comorbidities, inclusion and exclusion criteria, and blood test), potential confounder and outcomes. Participants who withdraw from our study for any reason will be followed up, and data will be analysed according to the intention-to-treat principle. All randomised participants will be followed up until 1year after randomisation.

\section{Data extraction and statistical analysis}

Data will be analysed on an intention-to-treat basis, including all participants enrolled in each group. Epidemiological and clinical characteristics data will be analysed using the $\chi^{2}$ test, non-parametric Kruskal-Wallis test and analysis of variance. Data presented in the text and tables will be reported as mean and SD, median and percentage (\%). Continuous variables will be analysed by using the paired t-test and the signed-rank test according to data distribution. $\mathrm{P}<0.05$ will be considered statistically significant. The software that will be used is SPSS for Windows, V.20.0 (IBM Corp., Armonk, New York, USA).

\section{Patient and public involvement}

Neither patients nor public were involved in the development of the research question, study design, outcome measures, recruitment to and conduct of the study or assessment of the burden of the intervention. The results of the study will be disseminated to study participants by means of lectures given by the investigators.

\section{DISCUSSION}

In this protocol, we described our randomised trial comparing the MAFRF to vaginal oestrogen and placebo in order to evaluate RF as a new treatment option for GSM. The strengths of this trial are the randomised design that allows control by confounding factors, ease in forming the control group and ability for the analysis of several clinical outcomes simultaneously. The main limitation is the loss of follow-up that occurs due to the extended treatment period. It is recognised that vaginal oestrogen may improve the symptoms of GSM. ${ }^{21} 28$

Some systematic reviews have already been published on the subject. ${ }^{29-32} \mathrm{~A}$ recent study assessing the physical methods for the treatment of GSM showed that, among physical methods, the $\mathrm{CO}_{2}$ laser continues to be one of the most commonly used methods, as it has the largest body of scientific evidence. The $\mathrm{CO}_{2}$ laser has been demonstrated to be an efficacious therapy for managing all GSM symptoms up to 12 months after treatment. ${ }^{32}$ The VHI score improved concerning elasticity, fluid volume, $\mathrm{pH}$, epithelial integrity and vaginal moisture, and VAS scores improved considerably for sensitivity, vaginal dryness, itching/stinging, dyspareunia and dysuria. The studies about the Er:YAG treatment showed that this method is effective, practical and safe too, and the effects are rapid and sustained for at least 12 months. Application of Er:YAG laser is associated with an improvement in vaginal atrophy, and such treatment induced a significant decrease in VAS, an increase of VHI and a substantial improvement in the UI. ${ }^{32}$

Additionally, the RF method could be a safe and effective non-surgical option for treating mild-to-moderate UI and other symptoms related to GSM. Significant improvements were observed in the mean VAS score and for complaints of VVA. However, little is known about the actual effectiveness of RF in the treatment of GSM/ UI since, as we have already reported in this review, the current literature is still sparse for this topic. For this reason, new research about this topic is necessary. ${ }^{32}$

We can also quote a prospective study ${ }^{28}$ conducted at a public university hospital to evaluate the effectiveness of MAFRF in the non-hormonal treatment of GSM. In this research, 55 postmenopausal women were examined 
before and after the treatment for VHI, vaginal microbiota, vaginal $\mathrm{pH}$ and cell maturation. The study observed an increase in the percentage of Lactobacillus spp after treatment. Consequently, there occurred a progressive decrease in vaginal $\mathrm{pH}$. Regarding cell maturation, there was a decrease in the percentage of parabasal cells and an increase in the rate of superficial cells. Additionally, there was an improvement in the VHI. In conclusion, the results showed that the therapy of MAFRF restored the vaginal balance, as would usually be expected with sufficient oestrogen levels. The predominance of Lactobacillus species and acidic $\mathrm{pH}$ of the vaginal fluid achieved after RF therapy could protect postmenopausal women from vaginal infections, inflammation and infections of the urogenital tract. Therefore, the MAFRF treatment was considered well-tolerated and promoted significant improvement in the vaginal microenvironment; therefore, RF could be an option for GSM symptoms. ${ }^{28}$

Although laser is the most well-known and used physical method, using RF presents advantages, such as the application is realised under direct vision and allowing the use of a vaginal speculum, facilitating treatment along the vaginal walls and preventing overlapping of shots. Also, the method is easy to learn and less costly. The procedure features a useful tolerance index, the patients recovered quickly and the microablation disappeared 3-5 days after the application.

\section{ETHICS AND DISSEMINATION}

All the procedures performed in this study involving human participants will be conducted in accordance with the ethical standards of the 1964 Declaration of Helsinki and its later amendments, the Declaration of Madrid of the World Psychiatric Association and the established requirements for manuscripts submitted to biomedical journals or comparable ethical standards of good clinical practice. The trial was approved by the local Division Ethics Committee of UFRN, under the number CAAE 81973618.2.0000.5292 (date of approval: 17 April 2018; reference number: CAAE 81973618.2.0000.5292) and was registered in REBEC (Brazilian Registry of Clinical Trials) before the enrolment of trial participants. Confidentiality of patients will be assured through data anonymisation.

Contributors ACAS and AKG were involved in drafting the study protocol. KSM and APFC were involved in statistical planning and drafting of the study protocol. JCC and FSF were involved in drafting and revising the study protocol. AKG developed the idea for this trial and was involved in drafting and revising the study protocol. ACAS conceived and designed the concept for this trial, was involved in drafting and revising the study protocol, and was the trial's principal investigator. All authors are involved in data acquisition and approved the final version of the manuscript.

Funding This work was supported by Brazilian CNPq (National Council for Scientific and Technological Development). Grant number 436740/2018-4.

\section{Competing interests None declared.}

Patient and public involvement Patients and/or the public were not involved in the design, or conduct, or reporting, or dissemination plans of this research.

Patient consent for publication Not required.

Provenance and peer review Not commissioned; externally peer-reviewed.
Open access This is an open access article distributed in accordance with the Creative Commons Attribution Non Commercial (CC BY-NC 4.0) license, which permits others to distribute, remix, adapt, build upon this work non-commercially, and license their derivative works on different terms, provided the original work is properly cited, appropriate credit is given, any changes made indicated, and the use is non-commercial. See: http://creativecommons.org/licenses/by-nc/4.0/.

ORCID iD

Ana Katherine Gonçalves http://orcid.org/0000-0002-8351-5119

\section{REFERENCES}

1 Management of symptomatic vulvovaginal atrophy: 2013 position statement of the North American menopause Society. Menopause 2013;20:888-902.

2 Portman DJ, Gass MLS, Vulvovaginal Atrophy Terminology Consensus Conference Panel. Genitourinary syndrome of menopause: new terminology for vulvovaginal atrophy from the International Society for the study of women's sexual health and the North American menopause Society. Maturitas 2014;79:349-54.

3 Gandhi J, Chen A, Dagur G, et al. Genitourinary syndrome of menopause: an overview of clinical manifestations, pathophysiology, etiology, evaluation, and management. Am J Obstet Gynecol 2016;215:704-11.

4 Tzur T, Yohai D, Weintraub AY. The role of local estrogen therapy in the management of pelvic floor disorders. Climacteric 2016;19:162-71.

5 Lethaby A, Ayeleke RO, Roberts H. Local oestrogen for vaginal atrophy in postmenopausal women. Cochrane Database Syst Rev 2016;8:CD001500.

6 Filippini M, Luvero D, Salvatore S. Efficacy of fractional CO2 laser treatment in postmenopausal women with genitourinary syndrome. Menopause 2019;27

7 Salvatore S, Nappi RE, Zerbinati N, et al. A 12-week treatment with fractional CO2 laser for vulvovaginal atrophy: a pilot study. Climacteric 2014;17:363-9.

8 Athanasiou S, Pitsouni E, Falagas ME, et al. $\mathrm{CO}_{2}$-laser for the genitourinary syndrome of menopause. How many laser sessions? Maturitas 2017;104:24-8.

9 Gambacciani M, Levancini M. Vaginal erbium laser as secondgeneration thermotherapy for the genitourinary syndrome of menopause: a pilot study in breast cancer survivors. Menopause 2017;24:316-9.

10 Flint R, Cardozo L, Grigoriadis T, et al. Rationale and design for fractional microablative $\mathrm{CO}_{2}$ laser versus photothermal non-ablative erbium:YAG laser for the management of genitourinary syndrome of menopause: a non-inferiority, single-blind randomized controlled trial. Climacteric 2019;22:307-11.

11 Vicariotto F, DE Seta F, Faoro V, et al. Dynamic quadripolar radiofrequency treatment of vaginal laxity/menopausal vulvovaginal atrophy: 12-month efficacy and safety. Minerva Ginecol 2017;69:342-9.

12 Casabona G, Presti C, Manzini M. Fractional ablative radiofrequency: a pilot study with twenty cases involving rejuvenation of the lower eyelid. Surg Cosmet Dermatol 2014;6:50-5.

13 Kamilos MF, Borrelli CL. New therapeutic option in genitourinary syndrome of menopause: pilot study using microablative fractional radiofrequency. Einstein 2017;15:445-51.

14 Chan A-W, Tetzlaff JM, Gøtzsche PC, et al. Spirit 2013 explanation and elaboration: guidance for protocols of clinical trials. BMJ 2013;346:e7586.

15 Schulz KF, Altman DG, Moher D, et al. Consort 2010 statement: updated guidelines for reporting parallel group randomised trials. BMJ 2010;340:c332.

16 Constantine GD, Simon JA, Pickar JH, et al. The REJOICE trial: a phase 3 randomized, controlled trial evaluating the safety and efficacy of a novel vaginal estradiol soft-gel capsule for symptomatic vulvar and vaginal atrophy. Menopause 2017;24:409-16.

17 Simon JA, Archer DF, Constantine GD, et al. A vaginal estradiol softgel capsule, TX-004HR, has negligible to very low systemic absorption of estradiol: efficacy and pharmacokinetic data review. Maturitas 2017;99:51-8.

18 Faubion SS, Sood R, Kapoor E. Genitourinary syndrome of menopause: management strategies for the clinician. Mayo Clin Proc 2017;92:1842-9.

19 Bachmann GA, Notelovitz M, Kelly SJ. Long-Term non-hormonal treatment of vaginal dryness. Clin Pract Sexuality 1992;8:3-8.

20 Sokol ER, Karram MM. An assessment of the safety and efficacy of a fractional $\mathrm{CO} 2$ laser system for the treatment of vulvovaginal atrophy. Menopause 2016;23:1102-7. 
21 Song S, Budden A, Short A, et al. The evidence for laser treatments to the vulvo-vagina: making sure we do not repeat past mistakes. Aust N Z J Obstet Gynaecol 2018;58:148-62.

22 Salvatore S, Nappi RE, Parma M, et al. Sexual function after fractional microablative $\mathrm{CO}_{2}$ laser in women with vulvovaginal atrophy. Climacteric 2015;18:219-25.

23 Thiel RdoRC, Dambros M, Palma PCR, et al. [Translation into Portuguese, cross-national adaptation and validation of the Female Sexual Function Index]. Rev Bras Ginecol Obstet 2008;30:504-10.

24 Hentschel H, Alberton DL, Capp E. Validation of the female sexual function index (FSFI) for Portuguese language. Rev. HCPA 2007;27.

25 Davis SR. Understanding female sexual function. Menopause 2009:3:425-6.

26 Ware J, Kosinski M, Keller SD. A 12-Item short-form health survey: construction of scales and preliminary tests of reliability and validity. Med Care 1996;34:220-33.

27 Bachmann GA, Nevadunsky NS. Diagnosis and treatment of atrophic vaginitis. Am Fam Physician 2000;61:3090-6.
28 Sarmento AC, Fernandes FS, Marconi C, et al. Impact of microablative fractional radiofrequency on the vaginal health, microbiota, and cellularity of postmenopausal women. Clinics 2020;75:1750.

29 Athanasiou S, Pitsouni E, Douskos A, et al. Intravaginal energy-based devices and sexual health of female cancer survivors: a systematic review and meta-analysis. Lasers Med Sci 2020;35:1-11.

30 Pitsouni E, Grigoriadis T, Douskos A, et al. Efficacy of vaginal therapies alternative to vaginal estrogens on sexual function and orgasm of menopausal women: a systematic review and metaanalysis of randomized controlled trials. Eur J Obstet Gynecol Reprod Biol 2018;229:45-56.

31 Jha S, Wyld L, Krishnaswamy PH. The impact of vaginal laser treatment for genitourinary syndrome of menopause in breast cancer survivors: a systematic review and meta-analysis. Clin Breast Cancer 2019;19:e556-62.

32 Sarmento ACA, Lírio JF, Medeiros KS. Physical methods for the treatment of genitourinary syndrome of menopause: a systematic review. IJGO 2020;153:200-19. 